\title{
Structural differences in the semantic networks of younger and older adults
}

\author{
Dirk U. Wulff ${ }^{1,2}$, Thomas T. Hills ${ }^{3}$, and \& Rui Mata ${ }^{1,2}$ \\ ${ }^{1}$ University of Basel \\ ${ }^{2}$ Max Planck Institute for Human Development \\ ${ }^{3}$ University of Warwick
}

\begin{abstract}
Cognitive science invokes semantic networks to explain diverse phenomena, from memory retrieval to creativity. Research in these areas often assumes a single underlying semantic network that is shared across individuals. Yet, recent evidence suggests that content, size, and connectivity of semantic networks are experience-dependent, implying sizable individual and age-related differences. Here, we investigate individual and age differences in the semantic networks of younger and older adults by deriving semantic networks from both fluency and similarity rating tasks. Crucially, we use a mega-study approach to obtain thousands of similarity ratings per individual to allow us to capture the characteristics of individual semantic networks. We find that older adults possess lexical networks with smaller average degree and longer path lengths relative to those of younger adults, with older adults showing less interindividual agreement and thus more unique lexical representations relative to younger adults. Furthermore, this approach shows that individual and age differences are not evenly distributed but, rather, are related to weakly-connected, peripheral parts of the networks. All in all, these results reveal the inter-individual differences in both the content and structure of semantic networks which may accumulate across the life span as a function of idiosyncratic experiences.
\end{abstract}

Keywords: semantic networks, cognitive aging, mental lexicon

\section{Introduction}

Semantic networks are the representational basis of our cognitive system (Baronchelli et al., 2013, Beer, 2000. Borge-Holthoefer \& Arenas, 2010) and an integral part of prominent models of memory (Anderson, 1983), reasoning (Collins \& Loftus, 1975), and creativity (Beaty et al., 2018; Kenett et al., 2018). Past work has often make the simplifying assumption that a common semantic network can be used to understand human semantic cognition (Anderson, 1983, Collins \& Loftus, 1975; Hills et al., 2012, Jones et al., 2015). This assumptions is implicit, for instance, in efforts to model retrieval from memory (Wulff et al., 2021), judgments of relatedness (Kraemer et al., 2021), or decision

Dirk U. Wulff (D) https://orcid.org/0000-0002-4008-8022 Thomas T. Hills (ID https://orcid.org/0000-0003-3842-2076 Rui Mata (I) https://orcid.org/0000-0002-1679-906X

We are grateful to Laura Wiles for editing the manuscript. This work was supported by a grant from the Swiss Science Foundation (100015_197315) to Dirk U. Wulff.

Correspondence concerning this article should be addressed to Dirk U. Wulff, Department of Psychology, University of Basel, Missionsstrasse 60-62, 4055 Basel, Switzerland. E-mail: dirk.wulff@gmail.com making (Bhatia, 2019) using large-scale word vector spaces and free-association networks. However, general theories of learning and development (Ramscar et al., 2014, Ramscar et al., 2017), as well as empirical findings (Benedek et al., 2017, Dubossarsky et al., 2017; Morais et al., 2013), suggest that semantic networks could vary considerably between individuals and across the life span. Crucially, researchers now seem to agree that understanding experience-dependent changes and individual variation in cognition is an important frontier for the science of aging (Lindenberger, 2014).

Aging research has made significant progress in the past decades quantifying age-related changes in semantic cognition, including large increases in the size of the knowledgestore across adult development, perhaps best documented in the large differences in vocabulary size in older relative to younger adults (Verhaeghen, 2003). More recently, however, research suggests that individual learning and life span development can also lead to changes in the structure of human knowledge (Cosgrove et al., 2021; Nation, 2017; Wulff et al., 2019). For example, recent efforts have used data from large scale free-association studies to show that older adults' semantic networks are less connected and efficient relative to those of younger adults (Dubossarsky et al., 2017, Wulff et al., 2021).

Quantifying individual and age differences in the size and 
structure of human knowledge is important because this may represent a missing link in understanding age-related decline in several aspects of cognitive functioning. Older adults tend to perform worse on a broad set of cognitive tasks, and such findings are commonly attributed to a decline in fluid cognitive abilities (Healey \& Kahana, 2016; Salthouse, 2010). However, some have argued that changes in the underlying size and structure of representations can contribute to age differences in cognitive performance, for example, due to activation-spreading across many targets in memory (fan effect; Buchler \& Reder, 2007)) or difficulties in discrimination learning between many similar items (Ramscar et al., 2017).

One first step needed to understand the contribution of semantic networks to age differences in cognitive performance is to document the changes in the size and structure of semantic networks across the life span. In the present study, we seek to describe potential life span differences in the structure of semantic networks by making two novel empirical contributions (see Figure 1). First, we investigate age differences in the size and semantic network structure for aggregates of younger and older groups obtained from verbal fluency data (e.g., "Name all animals you can") under different conditions: Our analyses of the semantic structure of verbal fluency productions are the first to include age comparisons using different categories (animal vs. country) to document whether structural differences generalize across domains; further, we investigate age differences in verbal fluency under different retrieval time allowances (1 minute vs. 10 minutes), which allow us to assess the idea that older adults can catch-up or even outperform younger adults in verbal fluency when given the opportunity to search their potentially larger semantic stores. Second, we adopt a megastudy approach (Keuleers \& Balota, 2018) to provide the first comparison of younger and older adults' semantic networks at the level of individuals in a study involving over 2,000 similarity ratings from each participant. This is crucial, given that aggregate networks likely do not accurately reflect the structure of individual level networks. Our work thus contributes to mapping the structural differences in the semantic networks of younger and older adults by considering different elicitation tasks (fluency, similarity) using both aggregate and individual semantic networks.

\section{Results}

\section{Age-related differences in fluency networks}

Verbal fluency is a neuropsychological test that requires participants to retrieve as many elements as possible from a natural category (Bousfield, 1953), say, animals, within in a given amount of time. Verbal fluency tasks are typically employed to measure fluid cognitive abilities, for instance, in screening instruments for age-related cognitive pathology

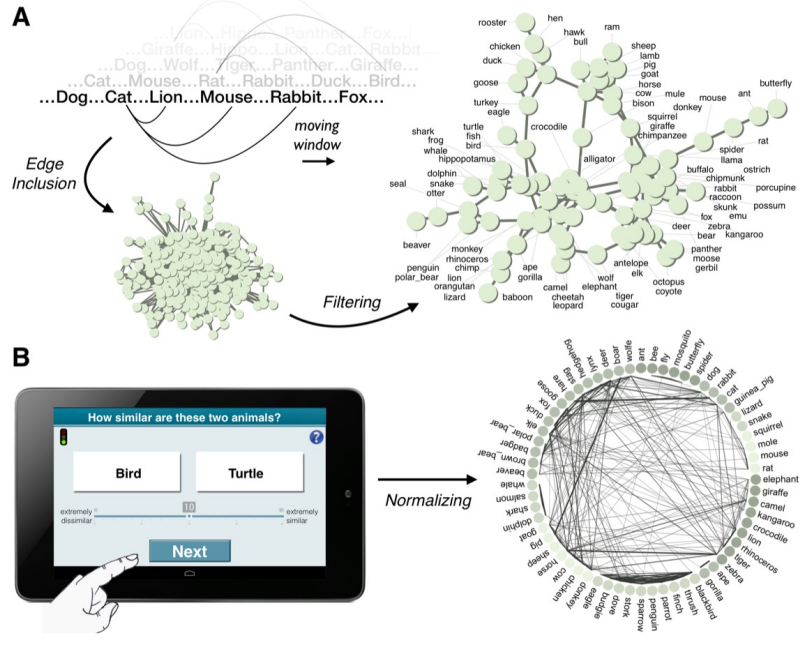

Figure 1

Methodological approach. Panel A illustrates the two steps, edge inclusion and filtering, involved in inferring networks from verbal fluency sequences. For details see Materials and Methods. The resulting network is based on 142 sequences of the older adults' group of study 1 . To simplify the visualization more conservative inferences settings were employed than used in the analyses reported below. Panel B illustrates the creation of networks from similarity ratings by normalizing individual's responses to the range of 0 and 1. The weighted network is based on the average ratings of the older adults' group of study 3 .

(e.g., Alzheimer's disease; Kalbe et al., 2004). In recent years, however, research has begun to analyze verbal fluency data in novel ways to extract from them semantic networks (Henry et al., 2004) and understand individual and age differences in semantic cognition (Cosgrove et al., 2021; Zemla \& Austerweil, 2018). Such approaches leverage the fact that the proximity of elements within the sequence of responses should reveal information on whether two elements are connected in an underlying semantic network. Several algorithms utilizing this principle have been proposed. To infer semantic networks of younger and older adults, we rely on a random walk plus filtering algorithm, which was recently found to predict human behavior better than other algorithms (Zemla \& Austerweil, 2018). In the first step, this algorithm adds to a single network for each age group edges for every pair of elements that occurred less than two positions apart from each other across all verbal fluency sequences of the age group. Then, in a second step, all edges in the network that were added only once across all sequences or were less frequent than expectation derived from random behavior are removed (see Figure 1A). Previous research has found this approach to produce plausible networks that predict human 
behavior better than other network inference methods for fluency data (Goñi et al., 2011; Zemla \& Austerweil, 2018).

We compared semantic networks of younger and older adults on the basis of four verbal fluency data sets, stemming from published work (Wulff et al., 2016) and two new studies (see Methods for details). Table 1 provides an overview of the datasets. Following previous work (Dubossarsky et al., 2017; Wulff et al., 2016; Zortea et al., 2014), we compared younger and older adults' networks with respect to three macroscopic network measures: average degree (connectivity, $\langle k\rangle$ ), average local clustering coefficient (structuredness, $C$ ), and average shortest path length (efficiency, $L$ ). These metrics are frequently employed to characterize the structure of cognitive networks and have been successfully linked to various measures of cognitive performance (for reviews, see Kenett et al., 2020; Siew et al., 2019; Wulff et al., 2019). For instance, degree has been linked to speed of retrieving words in lexical decison tasks (De Deyne et al., 2013). To avoid any confounding influences of network size, younger and older adults' networks were compared on the largest connected, common sub-graph, containing only words that were produced by both younger and older adults. Figure 2 shows the networks estimated for younger and older adults in each of the four data sets analyzed.

The results are presented in Figure 3. Compared to older adults, the networks of younger adults showed consistently higher average degrees and lower average shortest path lengths. However, results for the average clustering coefficient were mixed. A multiverse analysis (Steegen et al., 2016) evaluating the results under various implementations of our inference method suggests that inference is only robust for degree and shortest path length, but not for clustering, providing an explanation for the mixed results in the latter (see Supplementary Material). These results corroborate the existence of systematic structural differences between younger and older adults' semantic networks in terms of connectivity and efficiency, but not clustering.

Two additional findings concerning younger and older adults' verbal fluency data are worth noting. First, in the two studies that gave participants 10 minutes to retrieve items from semantic memory, there were no differences in the number of items produced by younger and older adults (Table 1). Compared with the shorter retrieval periods of previous studies (cf. Hills et al., 2013, Rosen, 1980, Tombaugh et al., 1999), the longer retrieval period of 10 minutes seems to eliminate older adults' disadvantage of slower memory retrieval. Second, as a group, older adults produced more unique category elements across all four data sets (Table 1), which is supportive of the notion that older adults possess a larger mental lexicon than younger adults (Verhaeghen, 2003). Despite such differences, the age-related patterns in macroscopic network structure generalize across the different domains and conditions, which speaks to the generality of
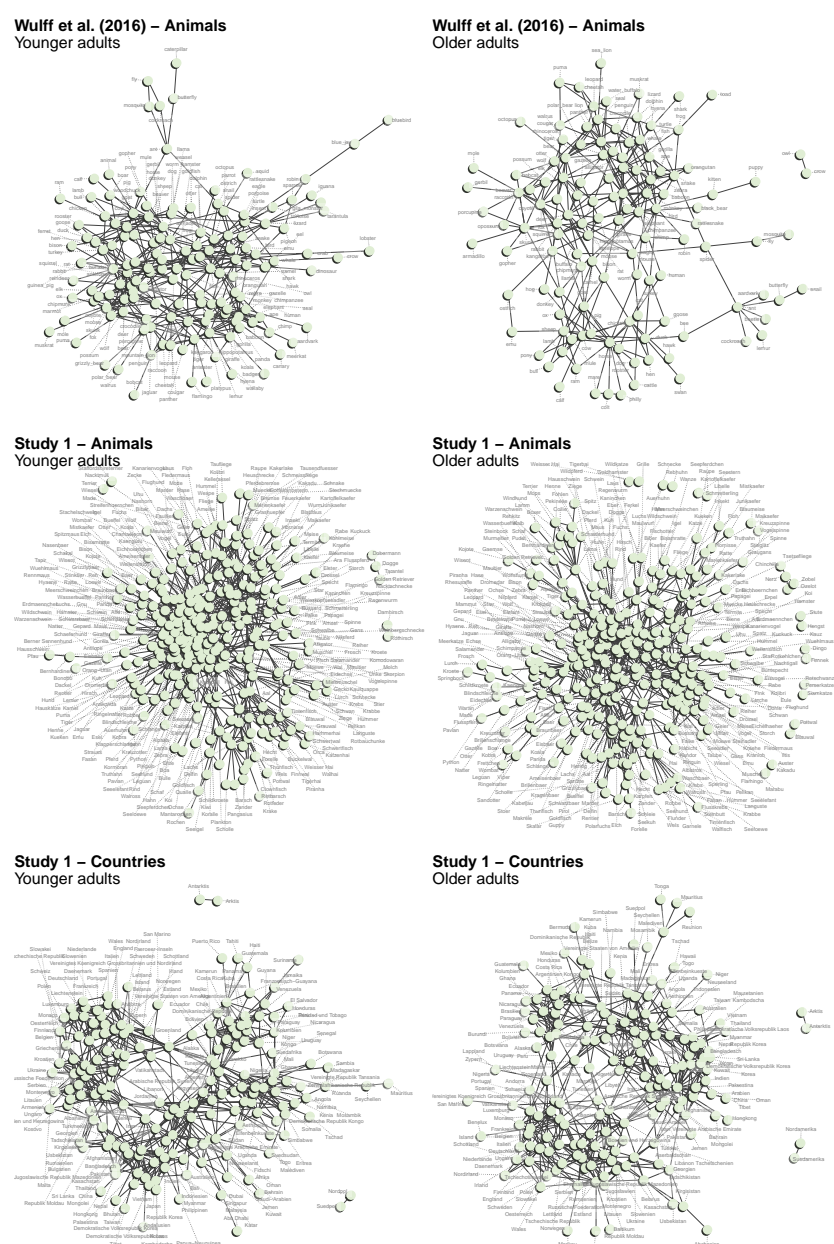

Study 1 - Countries
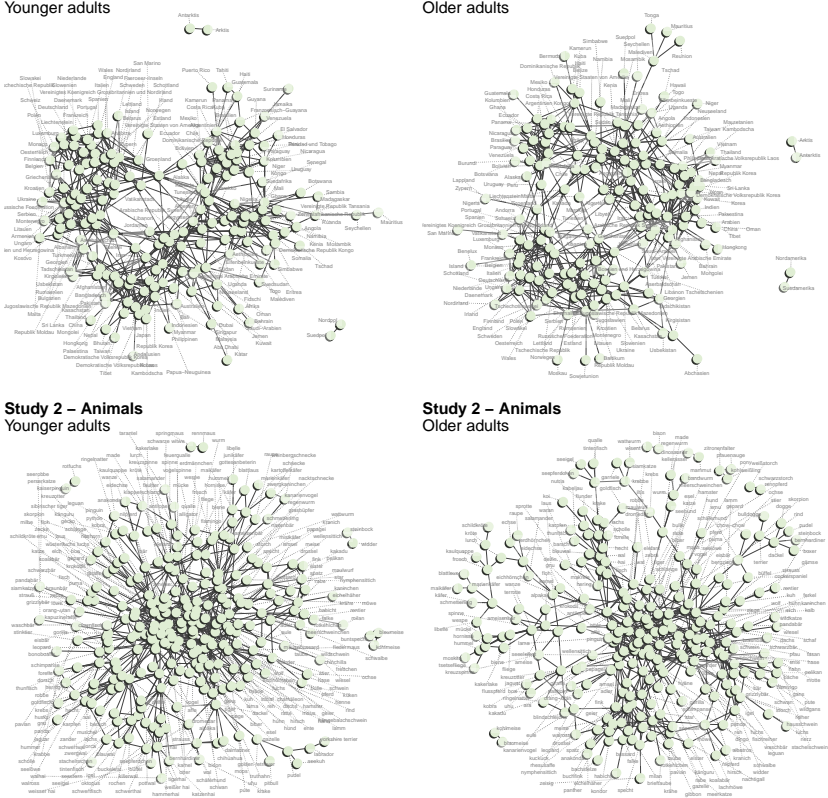

Figure 2

Fluency networks. Figure shows the networks estimated for younger (left column) and older adults (right column) in each of the four data sets analyzed. Labels are not displayed on top of their nodes to not obscure the structural characteristics of the network. For details on the network inference mechanism, see Methods. 


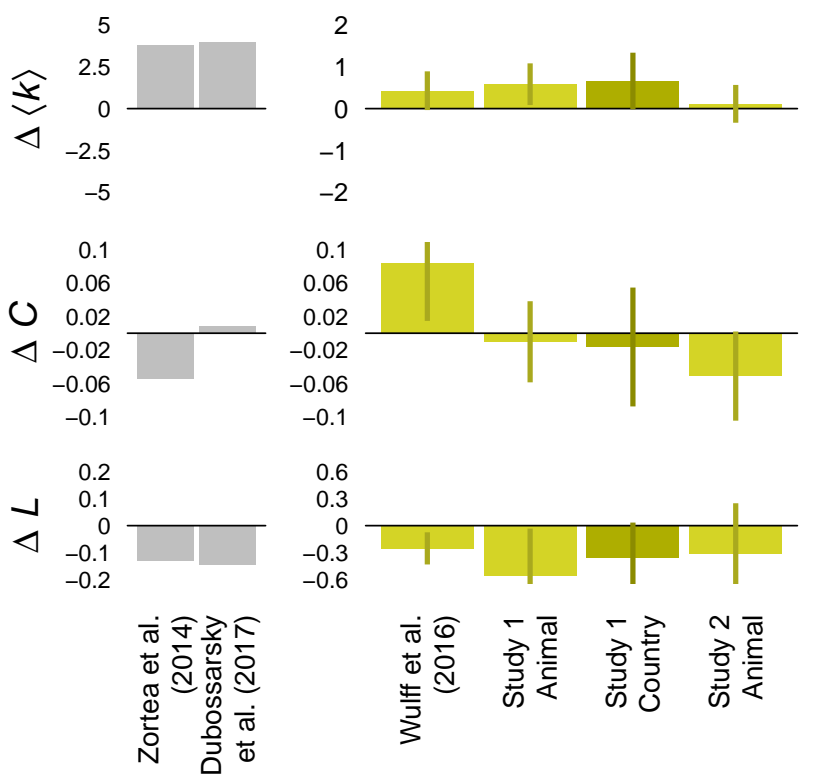

Figure 3

Differences in the macroscopic structure of younger and older adults fluency networks. Gray bars show the the difference between the younger and older adults' age group in Zortea et al. (2014) and that of age 30 and 70 in Dubossarsky et al. (2017), respectively. Yellow bars show differences in networks inferred from the four fluency data sets. Error bars show 95\% bootstrapped confidence intervals.

these findings across elicitation procedures (cf. Dubossarsky et al., 2017; Wulff et al., 2021).

\section{Age-related differences in individual-level similarity net- works}

A potential criticism of extant comparisons of younger and older adults' networks is that they lump together the data of many individuals to form aggregate networks, thus obscuring individual and group differences. To address this limitation, we conducted a comparison of younger and older adults' semantic networks at the level of the individual. Specifically, we elicited a large number of similarity ratings and constructed networks directly from each individual's responses. Aside from avoiding problems of aggregation, this approach had five additional advantages: First, similarity ratings likely recruit different memory retrieval processes and may overall be less affected by such processes than verbal fluency, permitting an independent and, potentially, cleaner assessment of network structure. Second, by requiring participants to rate a common set of words, similarity ratings likely are less affected by vocabulary differences between younger and older adults. Third, similarity ratings deliver direct estimates of the connection strength between words, sidestepping the need to infer edges using complex algorithms. Fourth, similarity ratings deliver graded responses permitting the construction of networks with weighted edges. Finally, because network statistics are available for each individual, the comparison between younger and older adults' networks can be carried out using standard methods of statistical inference.

In our study, each of 36 younger and 36 older participants provided a total of 2,253 similarity ratings, of which 1,953 were given to all possible pairs of 63 common animals and the remaining 300 to a set of repeat pairs, for which we found reliability to be high (older adults: $r=.76$, younger adults: $r=.74$ ). We constructed networks by, first, mapping an individual's ratings from the original scale of 1 (extremely dissimilar) to 20 (extremely similar) to the scale of 0 (minimum rating) to 1 (maximum rating), in order to account for differences in scale use. Second, we placed edges between all 63 animal nodes with weights equal to the transformed ratings. Finally, we eliminated edges with weights below a threshold $w_{\min }=[0, .1, .2, .3, .4]$. This last step was necessary to be able to determine the average local clustering coefficient, which is not defined for completely connected networks, while also providing us with a means to assess the robustness of our results to the choice of threshold. Figure 4 shows the 72 networks obtained from younger and older adults under $w_{\text {min }}=.1$.

Across all values of $w_{\min }$, compared to older adults, the networks of younger adults showed consistently higher average degrees $(\langle k\rangle)$ and lower average shortest path lengths $(L)$, and also higher local clustering coefficients $(C)$ (see Figure 5). We found the same pattern of results when the networks were analyzed as unweighted networks. For small values of $w_{\text {min }}$, where more than $50 \%$ of all edges were retained, i.e., $w_{\text {min }} \in(0, .1)$, moderate to large effects were observed that consistently reached statistical significance. Effects for more restrictive values of $w_{\min }$, i.e., $w_{\min }>.1$ pointed in the same direction, but they were smaller in size and, due to larger variance, did not consistently reach significance. These results corroborate the structural differences found for aggregate networks and demonstrate, for the first time, systematic age-related differences in the structure of semantic networks at the level of the individual.

Moreover, analyses reported in the Supplementary Material confirm the existence of aggregation biases. For the average degree, the clustering coefficient, and the average shortest path length, but not the average strength, estimates based on aggregate networks, which we derived by averaging networks within age groups, were considerably higher than the majority of estimates for individual-level networks. Aggregate networks, however, still revealed group differences consistent with those observed on the individual level, suggesting some level of robustness for comparisons of groups 


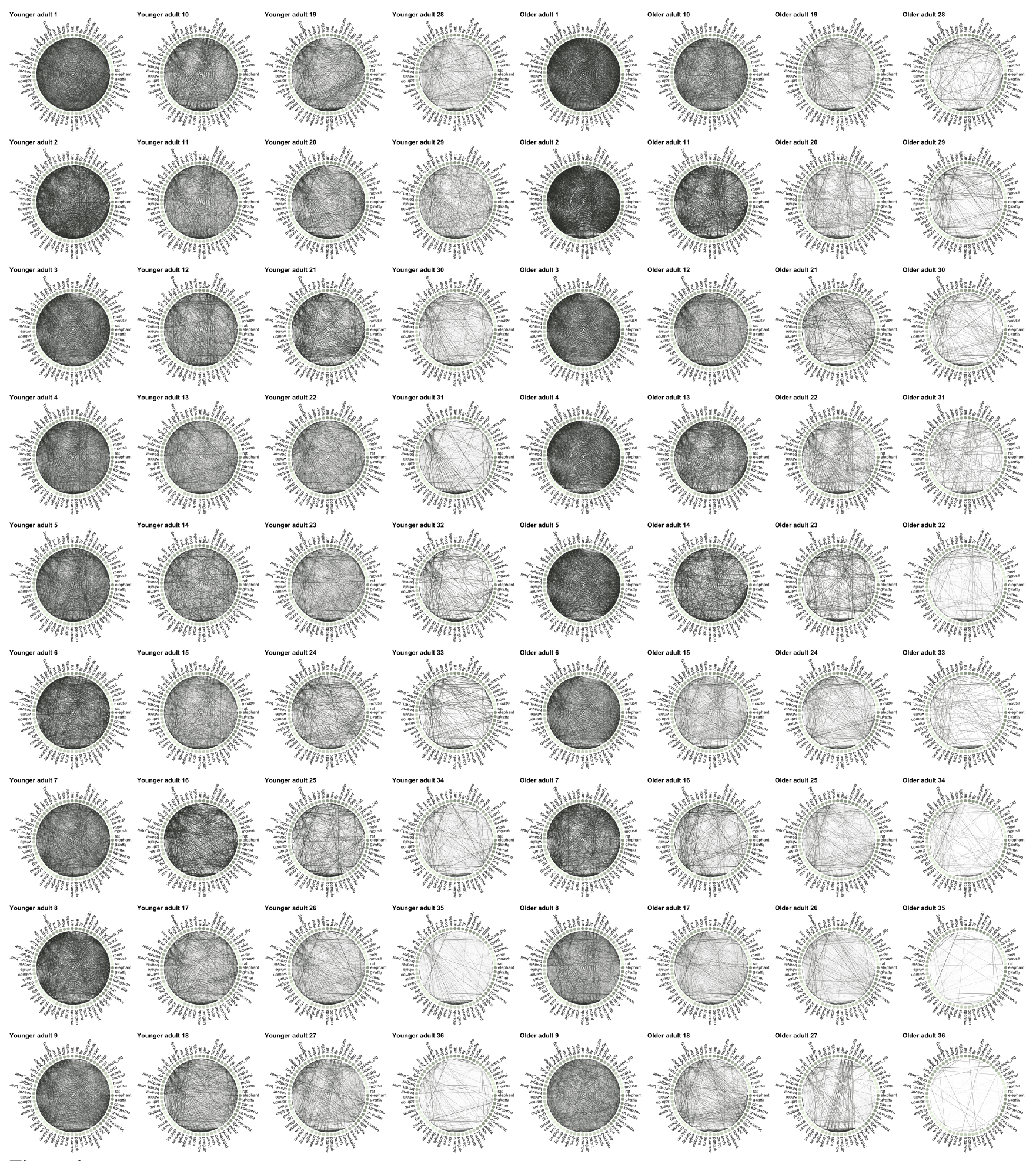

Figure 4

Similarity rating networks. Each individual plot shows the network of one individual under $w_{\text {min }}=.1$. The first four columns show, ordered by network strength, the networks of younger adults. The second four columns those of older adults. Edges weights have been scaled according to $w^{2}$ to increase visibility. Nodes are ordered and colored according to ten animal categories. These are, starting at $0^{\circ}$, African animals (plus kangaroo), large apes, birds, farm animals, fish, forest animals, pets, reptiles, and rodents. Animals names were translated from German. 
Table 1

An Overview of Fluency Data and their Inferred Macroscopic Network Structure

\begin{tabular}{ccccccccccc} 
Dataset & Age & $\mathrm{N}$ & $\mathrm{t}$ & $\bar{n}$ & $\bar{r}$ & $\frac{u}{\Sigma n}$ & $|V|$ & $\langle k\rangle$ & $C$ & $L$ \\
\hline Wulff et al. (2016) & $29-65$ & 142 & $1 \mathrm{~min}$ & 21.2 & .74 & $.09^{a}$ & 8 & $7.69^{c}$ & $.46^{c}$ & 2.53 \\
& $66-94$ & 142 & $1 \mathrm{~min}$ & 17.9 & .75 & $.11^{a}$ & 84 & $6.83^{c}$ & $.38^{c}$ & 2.78 \\
Study 1 - Animal & $18-34$ & 41 & $10 \mathrm{~min}$ & 90.7 & 2.71 & $.15^{a}$ & $209.1^{b}$ & $5.44^{c}$ & .18 & $3.51^{c}$ \\
& $66-81$ & 71 & $10 \mathrm{~min}$ & 89.6 & 12.6 & $.18^{a}$ & $209.1^{b}$ & $4.29^{c}$ & .13 & $4.07^{c}$ \\
Study 1 - Country & $18-34$ & 41 & $10 \mathrm{~min}$ & 75.3 & 2.41 & $.08^{a}$ & $150.9^{b}$ & $7.17^{c}$ & .19 & $3^{c}$ \\
& $66-81$ & 71 & $10 \mathrm{~min}$ & 69.7 & 10.9 & $.11^{a}$ & $150.9^{b}$ & $5.88^{c}$ & .21 & $3.36^{c}$ \\
Study 2 - Animal & $18-32$ & 36 & $10 \mathrm{~min}$ & 92.6 & 7.03 & .17 & 105 & 3.56 & $.33^{c}$ & 4.4 \\
& $65-78$ & 36 & $10 \mathrm{~min}$ & 88.6 & 11.4 & .19 & 105 & 3.33 & $.35^{c}$ & 4.72 \\
\hline
\end{tabular}

Note. ${ }^{a}$ Proportions were found to be significantly different between younger and older adults according to permutation tests. ${ }^{b}$ Bootstrap estimates. ${ }^{c}$ Significant $(p<.05)$ group difference according to bootstrap test.

Legend: $n$ - number of non-duplicate, valid responses; $r$ - number of duplicate responses; $u$ - Number of unique responses across the a group's retrieval sequences.

relative to one another.

\section{Locating Age-Related Differences in Semantic Network Structure}

Past work on the development of semantic knowledge suggests that cumulative linguistic experience and general learning process combine to create specific semantic structures that allow efficient discrimination learning (Ramscar et al., 2017). Crucially, that work proposes that such learning processes involve the strengthening of some associations while weakening others to allow differentiating between meaningful and meaningless pairs of items in memory. One important consequence of this process is that age differences in network structure may not be homogeneous across pairs of associations due to the interaction of learning and cumulative experience.

To shed light on the differences between younger and older adults' networks, we compared their networks on the level of node pairs with respect to three metrics that directly underlie the macroscopic results in Figure 5 and allow us to assess homogeneity of age differences across node pairs. Specifically, for each of the 1,953 node pairs, we compare the edge weight $w$ under $w_{\min }=0$ (corresponding to $\langle s\rangle$ and $\langle k\rangle)$, the proportion with which the pair forms triangles with other nodes $\left(C_{\text {pair }}\right)$ under $w_{\min }=.1$, and the path length connecting the pair $\left(L_{\text {pair }}\right)$ also under $w_{\min }=.1$. Figure 6 displays these results separately for younger and older adults with node pairs ordered by the average edge weight $w$ across both age groups. Ordering edges in this way allows direct inference-by-eye to reveal whether age-differences emerge uniformly across the network.

We observed consistent differences between younger and older adults in terms of all three metrics. Specifically, the edge weights and the proportion of triangles were consistently lower for older than younger adults, whereas path lengths were consistently larger. Crucially, we observed that the differences between older and younger adults were considerably larger for the lower half of node pairs. Thus, the differences between younger and older adults' networks appear to be mainly due to peripheral regions in the network, where edge weights are small, triangles rare, and shortest path lengths long.

We should note that the results above do not seem to be explained by age differences in use of the scale. We observed the judgments of younger and older adults not to differ in terms of the judged minimum $(d=0, p=1)$ or the judged maximum $(d=.26, p=.277)$. However, we did find younger and older adults to differ in terms of the ratings' average ( $d=.56, p=.019)$ and, crucially, the ratings' skewness $(d=-.51, p=.032)$, with older adults' ratings being lower on average and more right skewed. This suggests that younger and older adults interpreted and used the end points of the scales in the same way, and differed only in how they distributed the word pairs in between the end points, as would be expected from different perceptions of similarity between judged pairs.

\section{Assessing Age-Related Differences in the Similarity of Network Structure}

One corollary of the idea that cumulative experience is responsible for structural differences in semantic memory is not only that younger and older adults' semantic networks differ in key respects but, also, that older adults differ more from each other as a function of their different accumulated experiences (Wulff et al., 2019). We tested this principle by evaluating within age-group agreement in terms of edge 


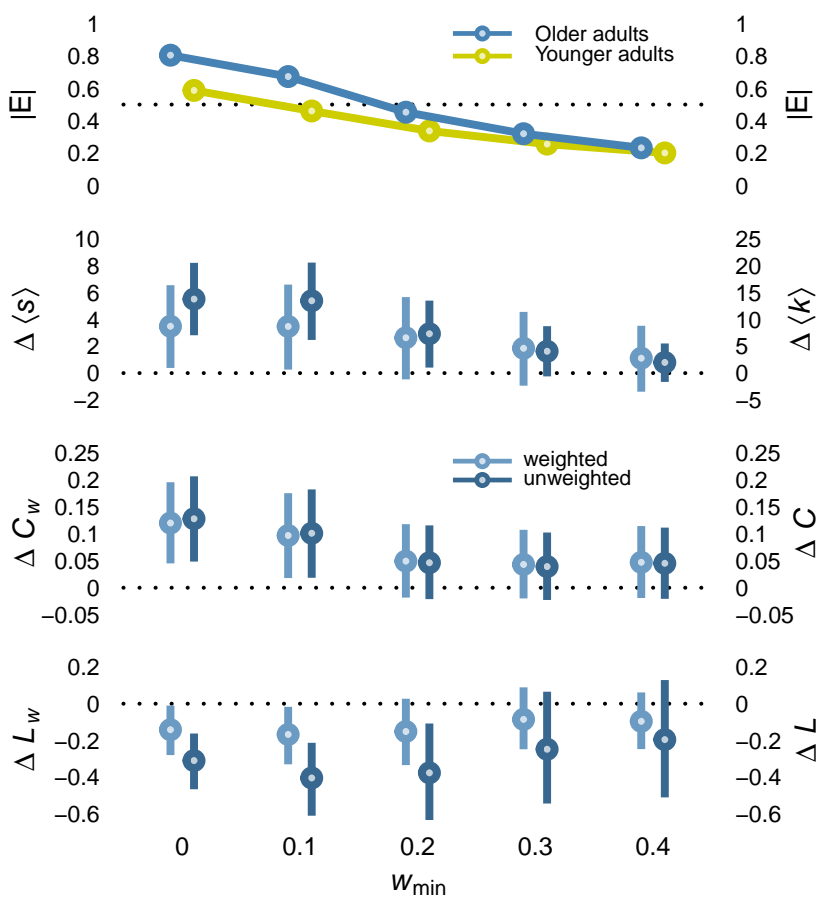

Figure 5

Differences in the macroscopic structure of younger and older adults' similarity rating networks. Blue and yellow circles, in panel 1, correspond to younger and older adults, respectively. In panels 2 to 5, light blue circles and dark blue circles correspond to differences between the younger and older adults' networks derived from weighted and unweighted networks, respectively. Error bars show 95\% bootstrapped confidence intervals.

weights. Specifically, we compared all pairs of individual networks using a weighted Jaccard index $(J I)$. We found older adults' networks to be considerably less similar to each other $(\overline{J I}=.33)$ than younger adults' networks $(\overline{J I}=.45$; $d=.97)$. This result is compatible with the idea that cumulative exposure to linguistic and other information contributes to individual differences in the structure of semantic networks.

\section{Discussion}

We investigated differences in the networks of younger and older adults at both the group and the individual level. Our group-level analyses using verbal fluency data replicate previously observed differences between networks of younger and older adults (e.g., Cosgrove et al., 2021; Dubossarsky et al., 2017; Zortea et al., 2014): The aggregate older adults' networks based on verbal fluency exhibited larger average degrees and lower average shortest path lengths than younger adults' networks, although the net-

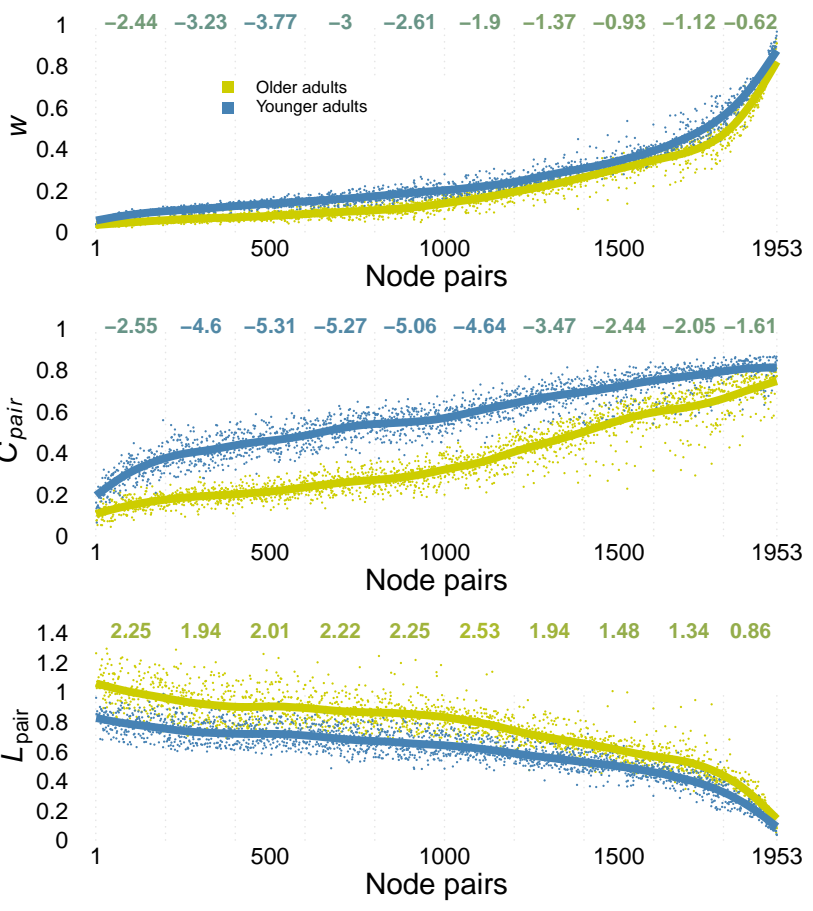

Figure 6

Comparisons between younger and older adults' networks across all 1,953 node pairs. The panels show separately for younger (blue) and older (younger) adults the average edge weights under $w_{\text {min }}=0$ (upper panel), the proportion of triangles that existing edges form with other edges under $w_{\text {min }}=.1$ (middle panel), and the shortest paths between the nodes $w_{\min }=.1$. The numbers on top of each panel show the Cohen's d (younger - older adults) for bins of 200 node pairs.

works of the two age groups did not systematically differ in their average clustering coefficients. Importantly, we extend past work by showing that these age patterns generalize across categories (animals, countries) and time constraints (1 vs. 10 minutes), suggesting that such age-related differences are not a function of specific elicitation choices and generalize across domains.

In addition, analyses of individual networks estimated from a similarity-judgment task involving thousands of judgments from the same individuals ruled out potential problems of aggregation and confirmed the differences in average degrees and lower average shortest path lengths, while additionally revealing systematic differences in terms of average clustering coefficients, in the direction of lower clustering in older adults' semantic networks. We found age differences were especially pronounced for weakly-related, peripheral regions of the network. Further, older adults' networks were shown to be considerably less similar to each other than 
younger adults' networks. All in all, these results provide converging evidence that the semantic networks of younger and older adults differ systematically not only in content, as has been amply suggested in past work (Verhaeghen, 2003), but also in their structure (Wulff et al., 2019). Our results are particularly novel in pointing out the progressively idiosyncratic nature of semantic representations across the life span, leading to more distinct semantic representations between individuals over time. Further, our finding that individual and age differences may be strongest for peripheral parts of semantic representations, emphasize the importance of investigating a large swath of individuals' semantic representations to understand the environmental and cognitive contributions to individual differences in semantic cognition.

We should point out a number of limitations in our work. First and foremost, we must acknowledge that we cannot definitively determine to what extent the age differences described above are due to age differences in representation and/or control processes involved in searching and selecting information from memory. The type of network models we adopt here to describe lexical associations are, in principle, compatible with mechanistic explanations based on both representation and process and, therefore, cannot fully arbitrate between the two (Castro \& Siew, 2020). Our finding that results generalize across elicitation conditions (time contraints), domains (animals, countries), and tasks (verbal fluency, similarity judgement) could be indicative of age differences being due to differences in the underlying representation, but only to the extent that one can confidently assume different processes of search and comparison across the different conditions, domains, and tasks. It seems plausible that the underlying cognitive processes are perhaps not identical but, at least similar, as all share aspects of controlled selection, involving the activation of concepts (e.g., "animal") and their features (e.g., "has wings"). There are two main approaches that could be interesting to further address the role of representation and process in engendering age differences in the semantic networks estimated from lexical tasks. One approach involves using additional independent measures to statistically account for the contribution of control processes using an individual differences approach (e.g., Hoffman, 2018). Another approach involves making use of neuroimaging techniques to directly measure mechanisms of control and memory retrieval. Past work suggests that representation and semantic control rely on distinct (but interacting) brain regions (Ralph et al., 2017) and this information could be potentially be leveraged to provide an estimate of the role of control processes in semantic cognition.

Second, on a related note, we do not detail a specific mechanism to account for the interaction between cumulative experience and network structure. Consequently, a key challenge for future research lies in developing models for the age-related changes in the structure of semantic networks reported here. One promising proposal stems from models of discriminative learning, whereby increasing experience leads weakly and strongly related contents in memory to be driven further apart from each other, resulting in a topological expansion of the network. The nature of structural differences, the observations of amplified differences for more weakly related words, as well as the lower similarity between older adults' compared to younger adults' networks, seem to support this notion. However, so far, discriminative learning has only been successfully employed to account for age differences in paired-associate learning (Ramscar et al., 2014, Ramscar et al., 2017). Whether such a mechanism can be expanded to account for the full set of results presented here remains an open question.

Third, and finally, our work made use of an extreme-group comparison design by comparing groups of younger relative to older adults. This type of design is not optimal to study the role of cumulative experience that is thought to underlie age differences in the content and structure of lexical and semantic networks. Ideally, estimates of cumulative experience and associated semantic networks would be obtained longitudinally for large samples of individuals and across long spans of time involving years or decades. One major difficulty with such studies will be mapping semantic networks for specific individuals but such efforts are under way (Wulff et al., 2021).

Despite its limitations, our work has some important implications for understanding and modeling human cognition. Both extant theories and some empirical evidence suggest sizable links between the structure of semantic networks and cognitive performance in a wider range of tasks (see Wulff et al., 2019, for a review). In many of these tasks, older adults are known to perform worse than younger adults (Salthouse, 2010), which is often considered a consequence of declining fluid abilities (Healey \& Kahana, 2016, Salthouse, 2010). Our and similar findings of systematic differences in semantic networks open up an alternative route leading to age differences in cognitive performance, whereby older adults' cognitive performance shows apparent decline because of the consequences of learning for the size and the structure of semantic networks. In turn, our finding that age differences may be particularly pronounced in peripheral parts of semantic networks could have implications for future tests of theories of individual and age differences in semantic cognition that may, or may not, make predictions concerning different parts of semantic representations.

Our results may have implications beyond our theoretical understanding of healthy cognitive aging. Lacking a cure, the best way to battle the "dementia epidemic" is timely diagnosis and early treatment (Larson et al., 2013; Robinson et al., 2015). The diagnosis of mild cognitive impairments and early dementia is, however, still predominately based on tests of cognitive performance (Robinson et al., 2015). Instru- 
ments such as the short dementia screener DemTect (Kalbe et al., 2004) or the neuropsychological battery CERAD (Fillenbaum et al., 2008) involve an individual undergoing a series of standard cognitive tasks, including several of the tasks listed above. Understanding the role of age-related changes in the structure of semantic networks promises to improve our interpretation of current instruments for dementia screening and diagnosis. Further research in this direction could lead to more personalized instruments that can detect changes in cognitive performance earlier and with higher sensitivity by focusing on specific parts of semantic representations than is currently done.

In sum, we presented converging results from verbal fluency and similarity judgment tasks concerning structural differences in the semantic networks of younger and older adults. Older adults seem to possess richer more idiosyncratic networks, characterized by smaller average degree and longer path lengths relative to those of younger adults. Our results emphasize the importance of considering how life span cognitive development and cumulative experience shape the content and structure of individuals' semantic cognition.

\section{Methods}

\section{Fluency data}

Four data sets from three studies were used to infer networks from fluency data. The first data set was obtained from (Wulff et al., 2016), who analyzed the data of two published studies, i.e., from Hills et al. (2013) and the Midlife in the United States (MIDUS3) longitudinal study. The data of Hills et al. (2013) contains three waves of responses to oneminute animal fluency task collected at Stanford University, CA. At time point one, the data included a total of 201 participants aged 27 to $99(\mathrm{Mdn}=68)$. To avoid practice effects and problems associated with participant attrition, we used only the first wave. The MIDUS3 data contained one-minute animal fluency data - recorded in phone interviews - from 104 individuals aged 34 to 83. Audio recordings were transcribed by us (see Supplementary Material). In order to obtained a sufficient amount of data to infer fluency networks, we joined the two data sets, but eliminated individuals with fewer than 10 fluency productions and mini-mental state values lower than 26, which is indicative of either low attention to the task or the onset of age-related disorders. Groups of younger and older adults were created by splitting the data at the median age. This resulted in groups of 142 individuals each aged 29 to 65 years old and 66 to 94 years old, respectively. Our first study with original data was collected in the context of another study on age-difference in decision making running in the laboratories of the Max Planck Institute (MPI) for Human Development, Berlin. We collected 10minute fluency data for both animals and countries from 71 older adults and 41 younger adults. Responses were recorded using a microphone and transcribed by us. Participants were recruited through the internal participant database of the MPI for Human Development. The older adults' age ranged from 65 to 80 years with a median age of 70 years, the younger adults' age ranged from 17 to 33 with a median age of 25 . Participants were paid $10 € /$ hour for participation. The second study was also collected at the Max Planck Institute for Human Development using participants from the MPI's internal database. We collected 10-minute fluency data for animals from 36 older adults and 36 younger adults. Responses were recorded using a microphone and transcribed by us. The older adults' age ranged from 65 to 78 years with a median age of 70 years, the younger adults' age ranged from 18 to 32 with a median age of 24 . Participants were paid $10 € /$ hour for participation. Study 1, 2 and 3 were approved by the internal review board of the Max Planck Institute for Human Development.

Fluency data were subjected to minimal preprocessing. Responses were scrutinized for category membership and spelling. A lenient criterion was used to assess category membership to retain as much of the original data as possible. In the case of animals, all nonfictional entries that described entire, nonhuman, and nonfictional animals were retained. This led us to exclude a few cases from the data, such as Godzilla, cat eye, or animal trainer. Similarly, in the case of countries, we retained all existing and named territories such as Istrien, a region of Italy, Croatia and Slovenia, the desert Sahara or cities, but not nonexisting, fictional territories such as Middle-earth. Spelling was hand-corrected on the basis of the Merriam-Webster online dictionary. Overall $96.8 \%$ to $99 \%$ of responses were retained in the analysis.

\section{Measures of macroscopic network structure}

The average degree of a network $G=(V, E)$, with nodes (or vertices) $V$ and edges $E$, is defined as $\langle k\rangle=\frac{2|E|}{|V|}$ for unweighted networks and as $\langle k\rangle=\frac{2}{|V|(|V|-1)} \sum_{i, j \in V ; i \neq j} a_{i j} w_{i j}$, where $a_{i, j}$ denotes the presence of an edge between nodes $i$ and $j$ and $w_{i, j}$ the according edge weight. The average degree or strength, as it is commonly referred to for weighted networks, describes the average connectivity in the network. The average local clustering coefficient for unweighted networks is defined as $C=\frac{1}{|V|} \sum_{i \in V} C_{i}$ with $C_{i}=\frac{2}{\left|k_{i}\right|\left(k_{i}-1\right)} \sum_{j, h \in N_{i}} a_{j h}$ and $k_{i}$ being the degree of node $i$ and $N_{i}$ the set of neighbors to $i$. For weighted networks, $C_{i}^{w}=\frac{1}{\left|s_{i}\right|\left(k_{i}-1\right)} \sum_{j, h \in N_{i}} \frac{w_{i j}+w_{i h}}{2} a_{i j} a_{i h} a_{j h}$ with $s_{i}=\sum_{j \in N_{i}} w_{j}$ being the strength of node $i$, the weighted analog to $k_{i}$. The local clustering coefficient describes the degree of transitivity in the network and is related to network modularity (Newman, 2006). It is often conceived of as an indicator of the structuredness of a network (Barrat et al., 2004). The average shortest path length is defined as $L=\frac{2}{|V|(|V|-1)} \sum_{i, j \in V ; i \neq j} L_{i j}$ where $L_{i j}$ is the length of shortest path between nodes $i$ and 
$j$, also known as the geodesic distance. For weighted networks, $L_{i j}$ is the sum of weights rather than the length. The average shortest path length describes the average distance between nodes. Low average shortest path lengths have been associated with efficient information processes (Bullmore \& Sporns, 2012; Latora \& Marchiori, 2001).

\section{Network inference approach}

Networks were inferred from verbal fluency data based on the community model developed by Goñi et al. (2011) and studied by Zemla and Austerweil (2018). The model is based on a two-step procedure. First, nodes and edges are included for every pair of responses that occurred within a distance of $l$ responses. For instance, for the response sequence "dog, cat, mouse, rabbit" and a criterion of $l=2$, edges would be included for all pairs less than three responses apart, excluding only the pair dog and rabbit, which are three responses apart. Second, an edge is identified as a true edge if the frequency of the connected words occurring with $l$ or fewer steps apart exceeded a frequency threshold $t_{\min }$ reflecting the required minimum frequency of co-occurring within $l$ responses to be considered in the first place, as well as a frequency threshold $t_{\text {chance }}$. The latter is derived from the probability $p_{i j}^{\text {linked }}$ of two words occurring within $l$ responses by chance, which is calculated as $p_{i j}^{\text {linked }}=p_{i} j^{\text {co-occur }} * p_{i j}^{\geq l}$. Furthermore, $p_{i j}^{\text {co-occur }}$, the probability of two words to co-occur within a fluency sequence, and $p_{i j}^{\geq l}$, the probability that two responses are no more than $l$ responses apart, are calculated as $p_{i j}^{c o-o c c u r}=\frac{f_{i} f_{j}}{M M}$ and $p_{i j}^{\geq l}=\frac{2}{N(N-1)}\left(-l N \frac{l(l+1)}{2}\right)$ with $f_{i}, f_{j}$ denoting the number of times two responses occur across $M$ sequence and $N$ denotes the average number of productions per sequence. $t_{\text {chance }}$ is then defined as the $1-\alpha$ quantile of the binomial distribution $B\left(M, p_{i j}^{\text {linked }}\right)$. Consistent with prior literature, we set $l=1, t_{\min }=1$, and $\alpha=1$ (Goñi et al., 2011, Zemla \& Austerweil, 2018) for our main analyses. In addition, we evaluate the robustness of the results in a multiverse analysis (Steegen et al., 2016) presented in the Supplementary Material.

\section{Similarity ratings}

Similarity ratings were collected in the context of study 3 and prior to participants completing the verbal fluency task. Participants took home a tablet to provide, over the course of roughly one week, on a scale from 1 to 20 , similarity ratings for 2,268 pairs of animals, consisting of each possible pair of 63 frequently occurring animals and 315 repeated pairs. The 63 animals were selected on the basis of the verbal fluency responses of study 2 in a manner that equated word frequency across younger and older adult age groups. See Supplementary Material. Reliability was found to be high in both younger and older adults with correlations of $r=.76$, $r=.74$ for younger and older adults, respectively. Participants were paid $10 € /$ hour for participation in the lab session and a flat fee of $44.1 €$ for providing the similarity ratings.

\section{References}

Anderson, J. R. (1983). A spreading activation theory of memory. Journal of verbal learning and verbal behavior, 22(3), 261-295.

Baronchelli, A., Ferrer-i-Cancho, R., Pastor-Satorras, R., Chater, N., \& Christiansen, M. H. (2013). Networks in cognitive science. Trends in Cognitive Sciences, 17(7), 348-360.

Barrat, A., Barthelemy, M., Pastor-Satorras, R., \& Vespignani, A. (2004). The architecture of complex weighted networks. Proceedings of the National Academy of Sciences, 101(11), 3747-3752.

Beaty, R. E., Kenett, Y. N., Christensen, A. P., Rosenberg, M. D., Benedek, M., Chen, Q., Fink, A., Qiu, J., Kwapil, T. R., Kane, M. J., et al. (2018). Robust prediction of individual creative ability from brain functional connectivity. Proceedings of the National Academy of Sciences, 201713532.

Beer, R. D. (2000). Dynamical approaches to cognitive science. Trends in Cognitive Sciences, 4(3), 91-99.

Benedek, M., Kenett, Y. N., Umdasch, K., Anaki, D., Faust, M., \& Neubauer, A. C. (2017). How semantic memory structure and intelligence contribute to creative thought: A network science approach. Thinking $\mathcal{E}$ Reasoning, 23(2), 158-183.

Bhatia, S. (2019). Predicting risk perception: New insights from data science. Management Science, 65(8), 3800-3823.

Borge-Holthoefer, J., \& Arenas, A. (2010). Semantic networks: Structure and dynamics. Entropy, 12(5), 1264-1302.

Bousfield, W. A. (1953). The occurrence of clustering in the recall of randomly arranged associates. The Journal of General Psychology, 49(2), 229-240.

Buchler, N. E., \& Reder, L. M. (2007). Modeling age-related memory deficits: A two-parameter solution. Psychology and Aging, 22(1), 104.

Bullmore, E., \& Sporns, O. (2012). The economy of brain network organization. Nature Reviews Neuroscience, 13(5), 336.

Castro, N., \& Siew, C. S. Q. (2020). Contributions of modern network science to the cognitive sciences: revisiting research spirals of representation and process. Proceedings of the Royal Society A: Mathematical, Physical and Engineering Sciences, 476(2238), 20190825-25.

Collins, A. M., \& Loftus, E. F. (1975). A spreadingactivation theory of semantic processing. Psychological Review, 82(6), 407. 
Cosgrove, A. L., Kenett, Y. N., Beaty, R. E., \& Diaz, M. T. (2021). Quantifying flexibility in thought: The resiliency of semantic networks differs across the lifespan. Cognition, 211, 104631.

De Deyne, S., Navarro, D. J., \& Storms, G. (2013). Better explanations of lexical and semantic cognition using networks derived from continued rather than single-word associations. Behavior research methods, 45(2), 480-498.

Dubossarsky, H., De Deyne, S., \& Hills, T. T. (2017). Quantifying the structure of free association networks across the life span. Developmental Psychology, 53(8), 1560.

Fillenbaum, G. G., van Belle, G., Morris, J. C., Mohs, R. C., Mirra, S. S., Davis, P. C., Tariot, P. N., Silverman, J. M., Clark, C. M., Welsh-Bohmer, K. A., et al. (2008). Consortium to establish a registry for alzheimer's disease (cerad): The first twenty years. Alzheimer's $\mathcal{E}$ Dementia, 4(2), 96-109.

Goñi, J., Arrondo, G., Sepulcre, J., Martincorena, I., de Mendizábal, N. V., Corominas-Murtra, B., Bejarano, B., Ardanza-Trevijano, S., Peraita, H., Wall, D. P., et al. (2011). The semantic organization of the animal category: Evidence from semantic verbal fluency and network theory. Cognitive Processing, 12(2), 183-196.

Healey, M. K., \& Kahana, M. J. (2016). A four-component model of age-related memory change. Psychological Review, 123(1), 23.

Henry, J. D., Crawford, J. R., \& Phillips, L. H. (2004). Verbal fluency performance in dementia of the alzheimer's type: A meta-analysis. Neuropsychologia, 42(9), 1212-1222.

Hills, T. T., Jones, M. N., \& Todd, P. M. (2012). Optimal foraging in semantic memory. Psychological Review, 119(2), 431.

Hills, T. T., Mata, R., Wilke, A., \& Samanez-Larkin, G. R. (2013). Mechanisms of age-related decline in memory search across the adult life span. Developmental Psychology, 49(12), 2396.

Hoffman, P. (2018). An individual differences approach to semantic cognition: Divergent effects of age on representation, retrieval and selection. Scientific Reports, $8(1), 42$.

Jones, M. N., Hills, T. T., \& Todd, P. M. (2015). Hidden processes in structural representations: A reply to abbott, austerweil, and griffiths (2015).

Kalbe, E., Kessler, J., Calabrese, P., Smith, R., Passmore, A., Brand, M. a., \& Bullock, R. (2004). Demtect: A new, sensitive cognitive screening test to support the diagnosis of mild cognitive impairment and early dementia. International Journal of Geriatric Psychiatry, 19(2), 136-143.
Kenett, Y. N., Beckage, N. M., Siew, C. S., \& Wulff, D. U. (2020). Cognitive network science: A new frontier.

Kenett, Y. N., Levy, O., Kenett, D. Y., Stanley, H. E., Faust, M., \& Havlin, S. (2018). Flexibility of thought in high creative individuals represented by percolation analysis. Proceedings of the National Academy of Sciences, 201717362.

Keuleers, E., \& Balota, D. A. (2018). Megastudies, crowdsourcing, and large datasets in psycholinguistics: An overview of recent developments. The Quarterly Journal of Experimental Psychology, 68(8), 14571468.

Kraemer, P. M., Wulff, D. U., \& Gluth, S. (2021). A sequential sampling account of semantic relatedness decisions.

Larson, E. B., Yaffe, K., \& Langa, K. M. (2013). New insights into the dementia epidemic. The New England Journal of Medicine, 369(24), 2275.

Latora, V., \& Marchiori, M. (2001). Efficient behavior of small-world networks. Physical review letters, 87(19), 198701.

Lindenberger, U. (2014). Human cognitive aging: Corriger la fortune? Science, 346(6209), 572-578.

Morais, A. S., Olsson, H., \& Schooler, L. J. (2013). Mapping the structure of semantic memory. Cognitive Science, 37(1), 125-145.

Nation, K. (2017). Nurturing a lexical legacy: reading experience is critical for the development of word reading skill. NPJ science of learning, 1-.

Newman, M. E. (2006). Modularity and community structure in networks. Proceedings of the National Academy of Sciences, 103(23), 8577-8582.

Ralph, M. A. L., Jefferies, E., Patterson, K., \& Rogers, T. T. (2017). The neural and computational bases of semantic cognition. Nature Reviews Neuroscience, $18(1), 42-55$.

Ramscar, M., Hendrix, P., Shaoul, C., Milin, P., \& Baayen, H. (2014). The myth of cognitive decline: Non-linear dynamics of lifelong learning. Topics in Cognitive Science, 6(1), 5-42.

Ramscar, M., Sun, C. C., Hendrix, P., \& Baayen, H. (2017). The mismeasurement of mind: Life-span changes in paired-associate-learning scores reflect the "cost" of learning, not cognitive decline. Psychological Science, 28(8), 1171-1179.

Robinson, L., Tang, E., \& Taylor, J.-P. (2015). Dementia: Timely diagnosis and early intervention. Bmj, 350, h3029.

Rosen, W. G. (1980). Verbal fluency in aging and dementia. Journal of Clinical and Experimental Neuropsychology, 2(2), 135-146. 
Salthouse, T. A. (2010). Selective review of cognitive aging. Journal of the International Neuropsychological Society, 16(5), 754-760.

Siew, C. S., Wulff, D. U., Beckage, N. M., \& Kenett, Y. N. (2019). Cognitive network science: A review of research on cognition through the lens of network representations, processes, and dynamics. Complexity, 2019.

Steegen, S., Tuerlinckx, F., Gelman, A., \& Vanpaemel, W. (2016). Increasing transparency through a multiverse analysis. Perspectives on Psychological Science, 11(5), 702-712.

Tombaugh, T. N., Kozak, J., \& Rees, L. (1999). Normative data stratified by age and education for two measures of verbal fluency: Fas and animal naming. Archives of Clinical Neuropsychology, 14(2), 167177.

Verhaeghen, P. (2003). Aging and vocabulary scores: A meta-analysis. Psychology and Aging, 18(2), 3329.
Wulff, D. U., De Deyne, S., Aeschbach, S., \& Mata, R. (2021). Understanding the aging lexicon by linking individuals' experience, semantic networks, and cognitive performance.

Wulff, D. U., De Deyne, S., Jones, M. N., Mata, R., Consortium, A. L., et al. (2019). New perspectives on the aging lexicon. Trends in Cognitive Sciences.

Wulff, D. U., Hills, T. T., Lachman, M., \& Mata, R. (2016). The aging lexicon: Differences in the semantic networks of younger and older adults.

Zemla, J. C., \& Austerweil, J. L. (2018). Estimating semantic networks of groups and individuals from fluency data. Computational Brain $\mathcal{E}$ Behavior, 1-23.

Zortea, M., Menegola, B., Villavicencio, A., \& Salles, J. F. d. (2014). Graph analysis of semantic word association among children, adults, and the elderly. Psicologia: Reflexão e Crítica, 27(1), 90-99. 